\title{
Subgraph Anomaly Detection in Financial Transaction Networks
}

\author{
Yulong Pei \\ Eindhoven University of Technology \\ y.pei.1@tue.nl \\ Werner van Ipenburg \\ Cooperatieve Rabobank U.A. \\ werner.van.ipenburg@rabobank.nl
}

\author{
Fang Lyu \\ Eindhoven University of Technology \\ f.lyu@tue.nl \\ Mykola Pechenizkiy \\ Eindhoven University of Technology \\ m.pechenizkiy@tue.nl
}

\begin{abstract}
Effective anomaly detection is crucial for the success of many AIbased solutions in the financial domain, including e.g. fraud detection and risk modeling. Identifying anomaly from financial transaction networks is one of the challenging tasks that can be cast as a special instance of anomaly detection in networks. Existing methods typically attempt to detect only node-level anomalies, and assume prior knowledge to extract representative features for identifying anomalies. However, there exist collective fraudulent behaviors at the level of subgraphs rather than individual node. A ring structure for money laundering and a tree structure for pyramid schemes would be common examples. Also, in practice it is difficult to decide which features are more representative beforehand. In this paper, we introduce SADE (Subgraph Anomaly DEtection) framework that addresses these needs. SADE consists of two steps: 1) role-guided subgraph embedding, and 2) subgraph anomaly detection. Our approach for learning the subgraph embeddings allows to preserve both the local structure of subgraphs and the global structure of entire network by making use of global roles and local connections of nodes. The learnt representation allows effective use of the state of art anomaly detection approaches. Our extensive experiments on synthetic and real-world financial transaction networks demonstrate the effectiveness of SADE in learning subgraph embeddings without requiring any prior knowledge and detecting anomalous subgraphs.
\end{abstract}

\section{CCS CONCEPTS}

- Computing methodologies $\rightarrow$ Learning latent representations; Neural networks; Anomaly detection.

\section{KEYWORDS}

Subgraph Anomaly Detection, Graph Embedding, Financial Transaction Networks

\section{ACM Reference Format:}

Yulong Pei, Fang Lyu, Werner van Ipenburg, and Mykola Pechenizkiy. 2020. Subgraph Anomaly Detection in Financial Transaction Networks. In ACM

Permission to make digital or hard copies of all or part of this work for personal or classroom use is granted without fee provided that copies are not made or distributed for profit or commercial advantage and that copies bear this notice and the full citation on the first page. Copyrights for components of this work owned by others than ACM must be honored. Abstracting with credit is permitted. To copy otherwise, or republish, to post on servers or to redistribute to lists, requires prior specific permission and/or a fee. Request permissions from permissions@acm.org.

ICAIF '20, October 15-16, 2020, New York, NY, USA

(C) 2020 Association for Computing Machinery.

ACM ISBN 978-1-4503-7584-9/20/10 . \$ \$15.00

https://doi.org/10.1145/3383455.3422548
International Conference on AI in Finance (ICAIF '20), October 15-16, 2020, New York, NY, USA. ACM, New York, NY, USA, 8 pages. https://doi.org/10. $1145 / 3383455.3422548$

\section{INTRODUCTION}

The problem of anomaly detection in financial domain attracts attention from both academia and industry because of its challenges and significant implications in a wide range of $\mathrm{AI}$ applications such as fraud detection and risk modeling [3]. The stream of financial transaction can be represented as a network, in which each node represents a customer, and two nodes are connected by an edge if there is at least once money transfer between these two customers. Identifying anomaly from such financial transaction networks can be cast as a special instance of anomaly detection in networks [10].

Existing machine learning approaches for anomaly detection in transaction networks commonly suffer from two major limitations: allowing only node-level anomaly detection and requiring prior knowledge to extract representative features for identifying anomalies. In practice, node-level anomaly detection can miss collective fraudulent behaviors including e.g. pyramid schemes that are often manifested in tree-like subgraph structures and money laundering manifested in a form of a ring or clique structures. On the other hand, it is difficult to predetermine upfront which features would be useful for anomaly detection, because financial transaction networks are complex and there structures are evolving over time. Furthermore, the fraud transaction behaviors are getting more complex and adverse and therefore are hard to define along with the online trading explosion. Employing network embedding approaches is considered to be a promising direction. The variety of node-level [14, 33, 34] and graph-level $[1,8,27]$ embedding methods can be used to map a node (or a graph) into a point in a latent space, in which its coordinate is seen as the embedding representation. However, neither of existing embedding methods can facilitate effective detection of anomalous subgraphs because 1) node-level approaches only preserve the local connection between pairs of nodes failing to capture the differences between subgraphs, and 2) graph-level approaches either assume the input is a set of separate graphs (in tasks of graph clustering and classification) or that the inter-community edges between subgraphs can be neglected meaning that again the global structural information is not captured in the network embedding. This can be illustrated with an example shown in Figure 1. There are four subgraphs in this toy network 1a, three of which are normal communities (in red, orange and green colors) and one has a tree-like structure (in blue color). In this toy network, the local structures are represented by communities shown in different colors. The global structures are reflected 


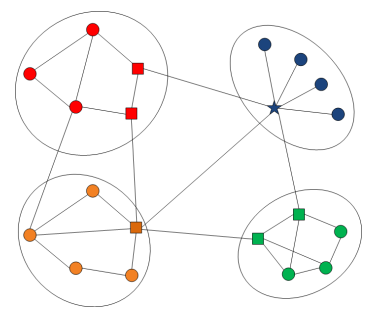

(a) Toy Network

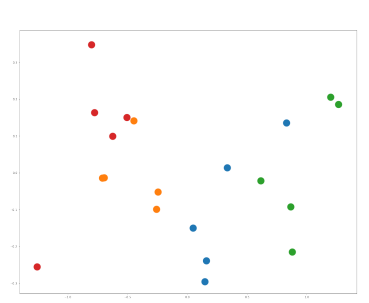

(b) Node-level embedding

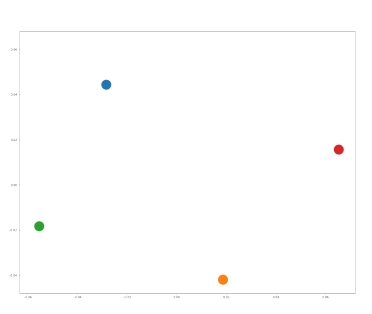

(c) Graph-level embedding

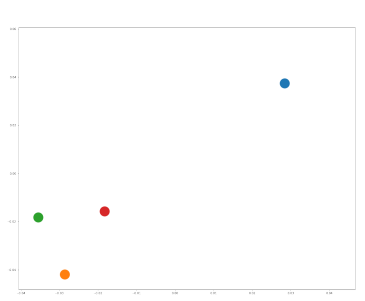

(d) Our method

Figure 1: An example of subgraph anomaly detection: (a) a toy graph with three normal subgraphs and one anomalous subgraph, (b) visualization of a node-level embedding method (node2vec [14]), (c) visualization of a graph-level embedding method (Sub2Vec [1]), and (d) visualization of our framework.

by roles, e.g., the pentagram node plays the role of as a star which is the center of the tree and the square nodes have the bridge role which connect different communities. Node-level embedding methods (Figure $1 \mathrm{~b}$ ) only preserves the local connections between node pairs resulting to failures in capturing the graph-level differences between normal and anomalous subgraphs. Existing graph-level embedding methods (Figure 1c) cannot preserve the global network structure and help in identifying complex (subgraph) structural outliers.

Therefore, we set our goal to design a graph-level embedding method which can preserve the local structure of nodes and global connection patterns of subgraphs simultaneously, to effectively distinguish normal and anomalous subgraphs combining those two kinds of complementary information. As shown in Figure 1d, in the latent space, normal subgraphs are close to each other while the anomalous subgraph is far away from the rest.

To achieve this goal, we propose a subgraph anomaly detection framework which consists of role-guided subgraph embedding and graph-level anomaly detection steps in turn. Role-guided subgraph embedding combines structural roles $[16,30]$ and random walk based embedding approach to learn subgraph embeddings. Thus, it is able to capture both global structures of the entire network and local structure of each subgraph. For the subgraph anomaly detection step, considering the trade-off between effectiveness and efficiency, we chose to employ Isolation Forest [24] to detect the anomalous subgraphs on the basis of the subgraph representations learned from previous step. Extensive experiments on several transaction networks have verified the effectiveness of our proposed framework on detecting anomalous subgraphs. Making use of the transfer relations, our proposed framework can serve as an early warning system at financial institutions. One can further explore the anomalous subgraphs for analyzing possible fraudulent or criminal behaviors.

Our main contributions are the following:

- We formulate the novel problem of subgraph anomaly detection in financial transaction networks (Section 2).

- We propose SADE: Subgraph Anomaly Detection framework to learn graph-level representations by taking into consideration of both local connections and global roles (Section 3).

- The results of our experiments on synthetic and real-world transaction networks demonstrate the effectiveness of SADE on subgraphs anomaly detection using different evaluation measures (Section 5).

\section{PROBLEM STATEMENT}

In this section, several necessary definitions are introduced and then the problem statement of the subgraph anomaly detection in transaction networks is presented.

Definition 1. Transaction Network. A transaction network $\mathcal{G}=\{V, E\}$ consists of: (1) a node set $V=\left\{v_{1}, v_{2}, \ldots, v_{|V|}\right\}$ representing customers/users in $\mathcal{G}$, where $|V|$ is the number of nodes; and (2) a edge set $E$ representing the money transfer relations between $V$, where $|E|$ is the number of edges.

For simplicity, we restrict our work here to undirected, unweighted networks and leave the extension to directed and weighted networks for future work.

Definition 2. Subgraph Embedding. Given $\mathcal{G}=\{V, E\}$ and an arbitrary subgraph set $\mathcal{S}=\left\{g_{1}, g_{2}, \ldots, g_{m}\right\}$ which is a partition of $\mathcal{G}$, Subgraph Embedding aims to represent each subgraph $g_{i} \in \mathcal{S}$ into a vector $W_{i}^{S} \in \mathbb{R}^{d}$ in a latent low-dimensional space $\mathbb{R}^{d}$, i.e., learning a mapping function $f: \mathcal{S} \rightarrow W^{S}$, where $W^{S} \in \mathbb{R}^{m \times d}$ and $d \ll|V|$. In the latent embedding space, both the global structural information of the input graph $\mathcal{G}$ and the local structural information of each subgraph $g_{i}$ can be preserved.

As introduced in the example in Figure 1a, roles provide a global view of networks and reflect structural functions nodes play in a network [28]. For example, nodes may function as the star, peripheral, clique or bridge. To preserve the global structural information for subgraph embedding, we propose a role-guided embedding approach which extracts structural roles to guide the embedding learning process.

Problem 1. Subgraph Anomaly Detection in Transaction Networks. Given $\mathcal{G}=\{V, E\}$ and an arbitrary subgraph set $\mathcal{S}=$ $\left\{g_{1}, g_{2}, \ldots, g_{m}\right\}$ which is a partition of $\mathcal{G}$, the task of subgraph anomaly detection is to find a subset of subgraphs that are most structurally different from the majority of subgraphs.

This is a general problem statement. Specifically, in our study, it is worth noting that

- We first use the role-guided subgraph embedding approach to map each subgraph into a feature vector representation. 
Thus, the problem is reduced to a traditional anomaly detection problem: calculating anomaly scores for all instances and then detecting anomaly by selecting the top ranked instances according to the anomaly scores.

- There are many possible ways to generate subgraph sets using different graph partition algorithms or domain knowledge. This makes the search space too large for real-world applications. Therefore, following previous studies $[1,6]$, we assume a set of subgraphs with graph theory or domainspecific knowledge based explanation is given as input.

\section{THE PROPOSED FRAMEWORK}

In this section we first introduce the proposed framework SADE, then we analyze the complexity of SADE. Overall, SADE consists of two steps: subgraph embedding and subgraph anomaly detection.

\subsection{Subgraph Embedding}

As stated in Section 1, the target of a desired framework for solving Problem 1 is twofold: (1) it captures both local structures from each subgraphs and global structures of the entire network, and (2) it does not require prior knowledge to extract representative features for anomaly detection. Towards this target, we propose a role-guided subgraph embedding method.

Compared to node embedding learning, it is more challenging to learn subgraph embeddings by preserving the structural information because there are two different types of structural information in this scenario:

- Local structures which are reflected by the intra-community edges. For instance, two connected nodes are expected to be similar to each other.

- Global structures which are reflected by the entire network including inter-community edges. For example, two stars in different subgraphs should be seen as similar.

Thus, a major challenge is to design an approach that has a global view of subgraphs, i.e., it has the capability to capture similarities and differences between the structures of subgraphs. To learn such representative embeddings of subgraphs, both kinds of structures should be preserved because they are complementary and neither could effectively distinguish subgraphs independently. For instance, it is difficult to quantify the similarities between two pyramid schemes with similar tree structure if only the local structures are preserved for representation learning. This results from that there may be no common nodes between these two subgraphs and using local structural information is impossible to find the similarity between them. On the other hand, one is unable to distinguish two core-periphery subgraphs without knowing the local structural connections, because the essential clues on the connection patterns between cores and peripheries are ignored.

To capture both local and global structures for subgraph embedding learning, we propose a new role-guided subgraph embedding framework $S A D E$, which combines the structural information from nodes' roles and co-occurrence between different subgraphs using random walk based embedding techniques. Because it has been shown that the roles of nodes capture their global structural similarities [16, 29] and random walk based embedding approaches preserves the local structures effectively $[14,33]$. Therefore, we take full advantages of those two components into solving 1 and propose our framework $S A D E$, which contains role discovery and embedding learning.

3.1.1 Stage 1: Role discovery. Structural roles represent the nodelevel connectivity patterns and functions that nodes play in a network. For example, a node may function as the center, peripheral, clique or bridge. Essentially, roles capture the global structural information of networks [28]. There are plenty of role discovery methods in previous studies using different theories, e.g., non-negative matrix factorization (NMF) [16], probabilistic graphical models [2], deep learning techniques [29]. Considering the trade-off between effectiveness and efficiency, we utilize RolX [16], a role discovery method using NMF, in this study.

RolX follows the feature-based role discovery framework which consists of role feature construction and role assignment. In detail, RolX firstly constructs a feature matrix $V_{n \times l}$ from the input network, where $n$ and $l$ are the number of nodes and features respectively. These features consist of local and egonet properties based on counts of links and the egonet-based properties generated in a recursive fashion [17].

Secondly, given the node-feature matrix $V_{n \times l}$, the idea of RolX is to find a rank $r$ approximation $G F \approx V$ where $r$ is the number of roles, matrix $G_{n \times r}$ denotes the nodes' affiliations to roles and matrix $F_{r \times l}$ represents the association of roles and features. Thus, the problem of role discovery is to seek two non-negative low rank matrices $G$ and $F$ to satisfy:

$$
\min _{G, F}\|V-G F\|_{F}^{2}, \text { s.t. } G \geq 0, F \geq 0
$$

where $\|\cdot\|_{F}$ is the Frobenius norm. The non-negativity constraint in Eq. (1) makes the representation of the original data easier to interpret and more semantically meaningful. Since this objective function is not convex for all the parameters $G$ and $F$ simultaneously, it is not feasible to find the global minimum. Multiplicative update rules [20] are used to find its approximate solution, showing as follows:

$$
G \leftarrow G \circ \frac{V F^{T}}{G F F^{T}}, F \leftarrow F \circ \frac{G^{T} V}{G^{T} G F}
$$

where $\circ$ denotes the element-wise product. For hard role assignment, node $i$ belongs to role $j$ if the $j^{\text {th }}$ element is the largest one in the $i^{t h}$ row of the nodes' membership matrix $G_{n \times r}$.

3.1.2 Stage 2: Embedding learning. After discovering roles which can capture the global structural information, the second stage is to learn subgraph embeddings. Similar to previous studies [1], we employ Paragraph Vector (PV) [19] to embed the graph-level similarities. PV learns latent representation of paragraphs and forces those paragraphs with high word co-occurrences to be mapped into embeddings with low distances. Obviously, PV considers the word level properties from both intra- and inter-paragraphs when producing paragraph level embeddings. Therefore, $\mathrm{PV}$ can capture both the local view of words inside the paragraph and the global view of the cross-paragraph information. By analogy with network data, subgraphs correspond to paragraphs and nodes in subgraphs are similar to words in paragraphs. The common characteristics of paragraph embedding and subgraph embedding make PV potential 


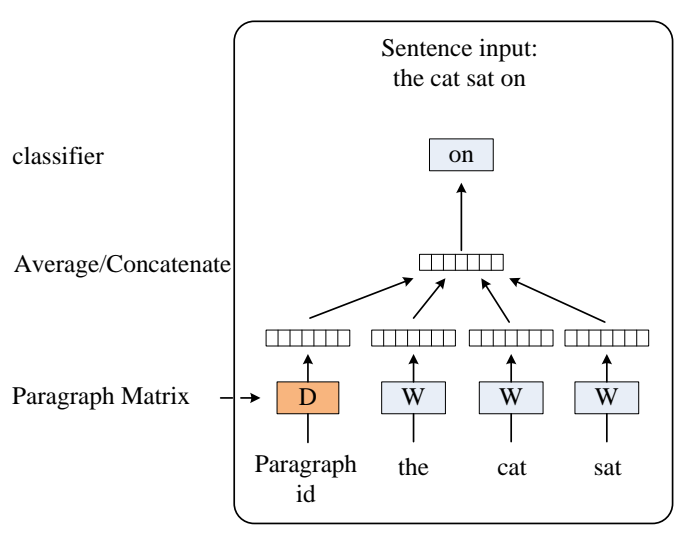

Paragraph2vec

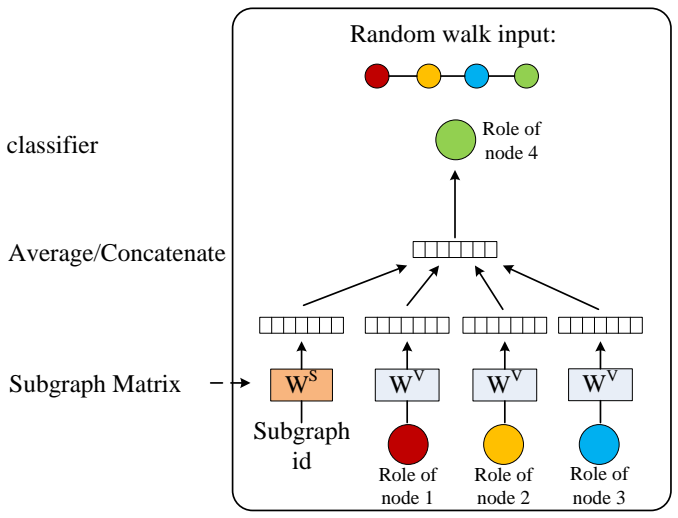

Subgraph Embedding

Figure 2: An illustration of extension from PV [19] to subgraph embedding. The left model is PV (adopted from [19]) and right one is our proposed subgraph embedding model. Note that the input for our model is role paths from truncated random walks.

in modeling global structures of subgraphs, which able to capture structural similarities and differences between subgraphs.

To extend PV to our task, we first perform truncated random walks [33] inside each subgraph $g_{i}$ separately, and not on the entire graph $\mathcal{G}$. After obtaining a set of paths, we transfer these node paths into role paths. For example, we have role assignment $r_{1}, r_{2}, r_{3}, r_{4}$ for nodes $n_{1}, n_{2}, n_{3}, n_{4}$ respectively. A node path $n_{1} \rightarrow n_{2} \rightarrow n_{4} \rightarrow n_{3}$ is transferred to role path $r_{1} \rightarrow r_{2} \rightarrow r_{1} \rightarrow r_{3}$. These role paths are inputted to the embedding process in PV. In the embedding process of PV framework (shown in Figure 2), each subgraph is mapped to a unique vector, represented by a column in subgraph embedding matrix $W^{S}$ and each node is also mapped to a unique vector, represented by a column in node embedding matrix $W^{V}$.

In the role-guided embedding, given the embedding $W_{i}^{S}$ of a subgraph $g_{i}$ and the embedding $W_{t}^{V}$ of a node $t$, the target is to predict the role $r$ of $t$. Formally the object of the embedding is:

$$
\max \sum_{g_{i} \in S} \sum_{t \in g_{i}} \log p\left(t \mid W_{i}^{S}, W_{t}^{V}\right)
$$

where $p\left(t \mid W_{i}^{S}, W_{t}^{V}\right)$ is the probability of predicting role of node $t$ given the vector representations of node $t$ and subgraph $g_{i}$. The prediction task is typically done via a multiclass classifier, e.g., softmax [19]. In this study, we follow previous studies to use softmax which is defined as:

$$
p\left(t \mid W_{i}^{S}, W_{t}^{V}\right)=\frac{\exp y_{t}}{\sum_{j} \exp y_{j}}
$$

Each of $y_{t}$ is un-normalized log-probability for each output node role $t$, which is defined as

$$
y_{t}=b+U h\left(W_{i}^{S}, W_{t}^{V}\right)
$$

where $U$ and $b$ are the softmax parameters. $h$ is constructed by a concatenation of subgraph embedding from $W^{S}$ and node embedding from $W^{V}$. After optimizing Eq (3), the output $W^{S}$ is the required subgraph embeddings.

\subsection{Subgraph Anomaly Detection}

Since we have learned the subgraph representation vectors in previous step, the target of subgraph anomaly detection is similar to traditional anomaly detection problems. In this paper, we employ Isolate Forest $[24,25]$ as the anomaly detection method because it has a low linear time complexity, requires a small memory, and can deal with high dimensional data.

Isolation Forest builds an ensemble of iTrees for a given data, the anomalies are those instances which have short average path lengths on the iTrees. The process of using Isolation Forest for anomaly detection consists of two stages: training stage which builds isolation trees using sub-samples of the given training set, and evaluation stage which passes test instances through isolation trees to obtain an anomaly score for each instance. In detail, the anomaly score $s$ for instance $x$ is defined as:

$$
s(x, \psi)=2^{\frac{-E((h(x))}{c(\psi)}}
$$

where $E(h(x))$ is the average of $h(x)$ from a collection of the constructed iTrees. $c(\psi)$ is the average of $h(x)$ given $\psi \cdot \psi$ is used to normalize $h(x)$ and defined as

$$
c(\psi)= \begin{cases}2 H(\psi-1)-2(\psi-1) / n & \text { for } \psi>2, \\ 1 & \text { for } \psi=2, \\ 0 & \text { otherwise }\end{cases}
$$

where $H(i \cdot)$ is the harmonic number and it can be estimated by $\ln (i)+0.5772156649$ (Euler's constant). $\psi$ is the subsampling size.

\subsection{Computation Complexity}

The complexity of two steps of SADE are analyzed as follows:

1 Subgraph embedding. There are two stages in this step. For the role discovery, the time complexity is linear on the number of edges, i.e., logarithm number of edges and formally it is $O(|E| \cdot f+|V| \cdot f \cdot r)$ [16], where $f$ is the number of extracted features and $r$ is the number of roles. For the embedding, using Hierarchical Softmax and Negative Sampling, 
the time complexity is $O(\log (|V|+|\mathcal{S}|)$, i.e., logarithm of the numbers of nodes and subgraphs.

2 Subgraph anomaly detection. In Isolation Forest based anomaly detection, the worse case time complexity of the training stage and the evaluation stage are $O\left(|T| \cdot \psi^{2}\right)$ and $O(|\mathcal{S}| \cdot$ $|T| \cdot \psi)$ respectively, where $|T|$ is the number of trees [25].

Overall, the framework is efficient which can be seen to be linear on the number of edges, as in practice the other parameters, e.g., the number of extracted features, roles, trees and the sub-sampling counts, are set to be much smaller values compared to the number of edges and nodes in large real-world networks.

\section{EXPERIMENTS}

In this section, we evaluate the effectiveness of our proposed SADE framework empirically. We first introduce experimental settings. Then we discuss our experimental results in different datasets and analyze the influence of parameters.

\subsection{Experimental Setup}

To demonstrate the effectiveness of our proposed framework in detecting anomalous subgraphs, we select several representative graph-level embedding methods as our baselines:

- graph2vec [27] constructs Weisfeiler-Lehman tree features for nodes in graphs and then learn graph representations by decomposing the graph-feature co-occurrence matrix.

- NetLSD [35] extracts compact graph signatures based on the heat or wave kernel of the Laplacian, which inherit the formal properties of the Laplacian spectrum.

- Gl2vec [8] extends graph2vec by exploring the line graph. The learned embeddings are generated by concatenating the embeddings of the original graph and line graph.

- FGSD [36] learns graph-level representations based on the family of graph spectral distances. The Moore-Penrose spectrum of the normalized Laplacian is used as the graph embedding.

- GeoScattering [11] uses geometric scattering transform as a deep filter bank for feature extraction. Moments of the wavelet transformed features are used as embedding.

- Sub2Vec [1] also utilizes PV model [19]. It extends PV to learn subgraph embedding while preserving distance between subgraphs that have similar 'node co-occurrences'.

Besides, we also compare SADE to an node-level embedding method, i.e., node2vec [14]. Note that we average the node embedding in each community as the subgraph representation.

\subsection{Ring, Star and Tree Detection in the Synthetically Generated Toy Network}

We use a toy example of the synthetically generated network to demonstrate the effectiveness of our proposed SADE framework in subgraph anomaly detection and the failure of other state-of-theart network embedding techniques in this task. The toy network consists of 10 subgraphs and each subgraphs has 10 nodes. There are 7 normal subgraphs following the power-law distribution of degrees and three anomalous subgraphs including 1 ring, 1 star and 1 tree with a depth of 3 .
Figure 3 shows the visualization of learned subgraph embeddings by different methods after using PCA to reduce the dimension to 2 . For our framework, the number of role is 16 . The latent dimension is 16 for all methods except methods that determine latent dimension automatically, i.e., NetLSD, FGSD and GeoScattering. For shared parameters in all methods, we use the same settings: number of walks per node: 20 , walk length: 64 , skipgram window size: 4 . Other parameters are set by default. It can be observed that most previous network embedding approaches can only detect part of these anomalous subgraphs and fail to identity all three different structures. For example, graph2vec, node2vec and GeoScattering only detect the star. Sub2Vec distinguish the ring and star but fail to identify the tree. However, our proposed framework can effectively detect all three anomalous subgraphs shown 3 i.

\subsection{Pyramid Scheme Detection from Bank Transactions}

In this experiment, we test the effectiveness of SADE in a real-world application, detecting pyramid schemes from a transaction network. Pyramid scheme is a kind of anomalous financial groups, in which each member's transaction behaviors could be normal, however collective behaviors of the whole group are fraudulent. In general, money is transferred from bottom accounts to top accounts, making the transaction subgraph inside each group in the shape of a tree. The target is to detect such anomalous pyramid schemes from a transaction network.

We use a real-world data consists of bank transaction records from a security institution in China. It contains 10141 bank accounts, connected by 33754 edges, belonging to 78 non-overlapping pyramid communities. The number of nodes in those anomaly subgraphs ranges from 89 to 198 . However, the information of normal accounts is unknown. Therefore, we derive and augment two networks based on the real-world data. Specifically, in the augmented networks anomaly subgraphs are tree-like structures from the real pyramid schemes, and normal subgraphs are communities whose size and node degrees obey power law distributions. Random edges are created between subgraphs.

We augment two networks for the experiment, a smaller network with 4 anomalous subgraphs (Small Network) and a larger network with 78 subgraphs (Large Network). A brief statistics of these networks is shown in 1 . Considering the characteristics of real case scenario in anomaly detection, we make the ratio between numbers of normal and anomalous subgraphs nearly 10:1. The experimental results are shown in Table 2 and 3. Note that for fair comparison, Isolation Forest has been used for all methods for anomaly detection. Under the evaluation measures of precision and recall, it can be observed that our proposed framework outperforms the-stateof-art approaches in detecting anomalous subgraphs, i.e., pyramid schemes. Since the Small Network is augmented from a subset of the real data, it is less complex than the Larger Network. Thus, the performance on the Small Network looks better.

\subsection{Parameter Sensitivity}

There are three parameters in our proposed SADE framework: (1) number of roles $r$, (2) latent dimension $d$ and (3) random walk length $l$. In the experiments, we investigate the impact of these parameters 
Table 1: Statistics of derived and augmented networks.

\begin{tabular}{ccccc}
\hline Dataset & Number of nodes & Number of edges & Number of normal subgraphs & Number of anomalous subgraphs \\
\hline Small Network & 6,000 & 28,065 & 42 & 4 \\
Large Network & 21,141 & $1,355,790$ & 657 & 78 \\
\hline
\end{tabular}

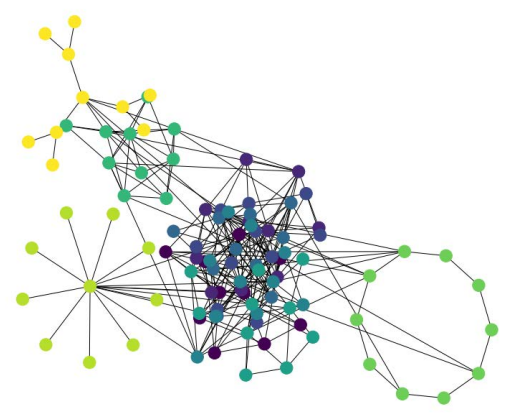

(a) Toy network

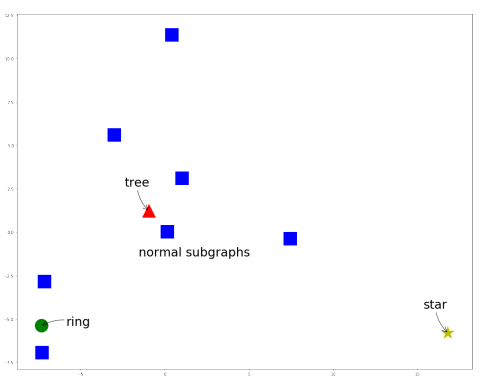

(d) node2vec

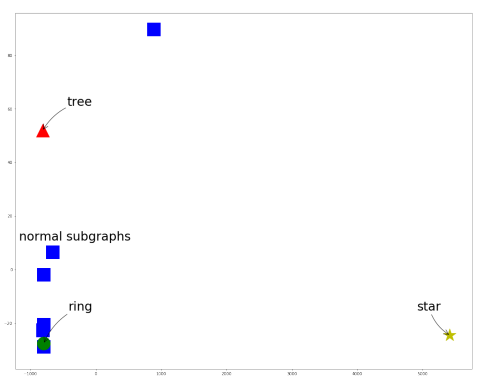

(g) GeoScattering

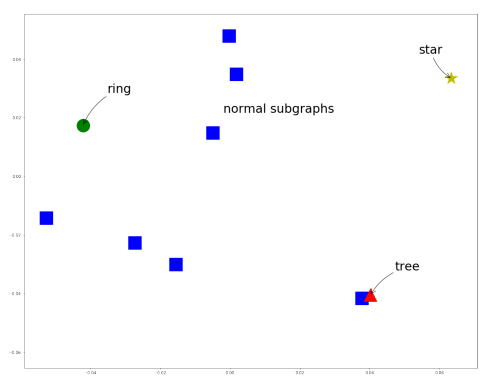

(b) graph2vec

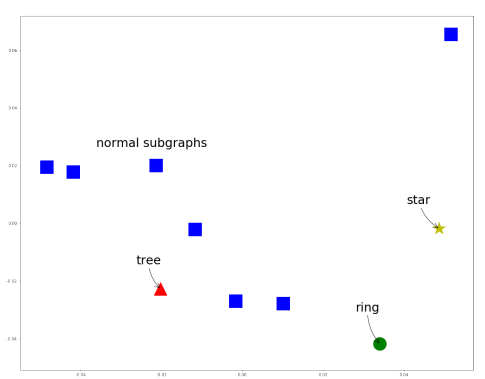

(e) Gl2vec

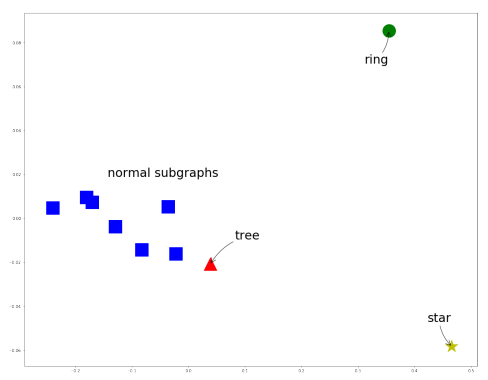

(h) Sub2Vec

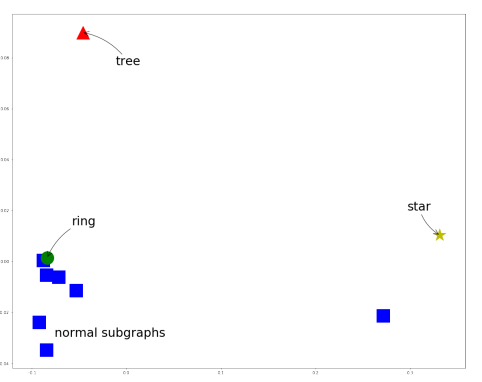

(c) NetLSD

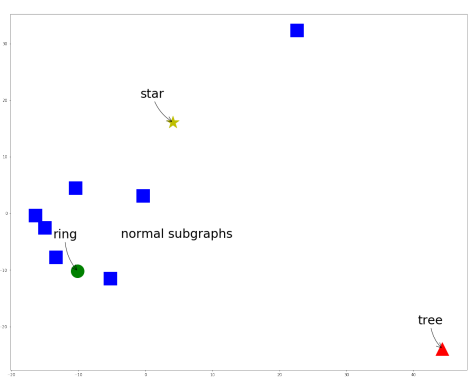

(f) FGSD

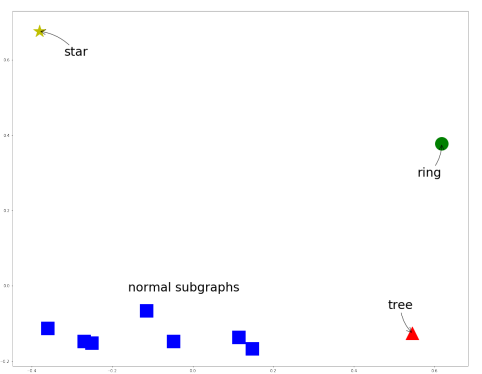

(i) Rupture

Figure 3: (a) The toy network and Visualization of subgraph embeddings learned by (b) graph2vec, (c) NetLSD, (d) test, (e) Gl2vec, (f) FGSD, (g) GeoScattering, (h) SUb2Vec, and (i) Our proposed method.

separately. Specifically, we test anomaly detection performances by ranging $r$ from 6 to 16 with interval $2, d$ from $2^{4}$ to $2^{9}$, and $l$ from $2^{3}$ to $2^{8}$. The results for different parameters are shown in Figure 4

From the results, it can be observed that the impacts of different parameters are different. Some conclusions on parameter selection can be drawn: (1) larger role numbers generally achieve better performance because larger values can capture the differences in global roles; (2) latent dimensions should not be too small or too large because smaller dimension may lack representation power while larger value may contain redundant information; and (3) random walk length also should not be too small or too large with similar reasons to dimension.

\section{RELATED WORK}

Our research is related to two lines of studies: anomaly detection in networks and graph embedding. 


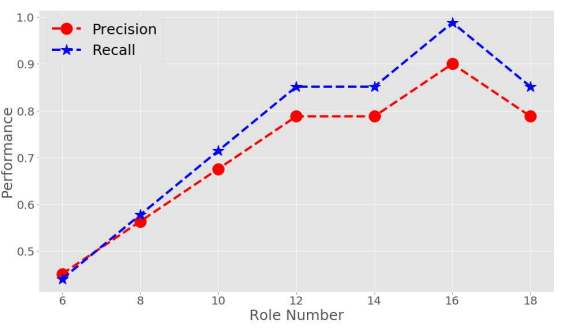

(a) Influence of Role numbers

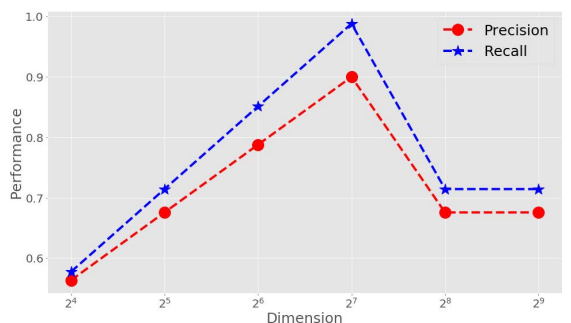

(b) Influence of latent dimensions

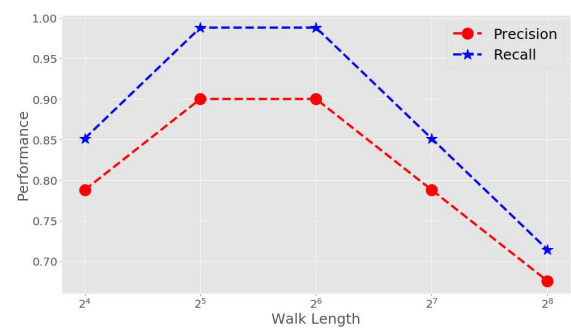

(c) Influence of walk length

Figure 4: Analysis of sensitivity of different parameters in Small Network.

Anomaly Detection in Networks. Anomaly detection is one of the most important research questions in different types of data, e.g., text [18] and networks [5]. Compared to anomaly detection on other types of data, anomaly detection in networks is challenging because network data disobeys the i.i.d. In this section, we focus on the related works of anomaly detection in networks.

CODA [12] focuses on community anomalies by simultaneously finding communities as well as spotting anomalies using a unified probabilistic model. AMEN [32] uses both attribute and network structure information to detect anomalous neighborhoods. Radar [21] detects anomalies whose behaviors are singularly different from the majority by characterizing the residuals of attribute information and its coherence with network information. ANOMALOUS [31] is a joint anomaly detection framework to optimize attribute selection and anomaly detection using CUR decomposition of matrix and residual analysis on attribute networks. DOMINANT [9] utilizes GCN to compress the input attributed network to succinct low-dimensional embedding representations and then reconstruct both the topological structure and nodal attributes with these representations. MADAN [15] is a multi-scale anomaly detection method. It uses the heat kernel as filtering operator to exploit the link with the Markov stability to find the context for outlier nodes at all relevant scales of the network.

Graph Embedding. Graph embedding methods aim to map nodes or graphs into points in a low-dimensional space according to their structural information in the given graph(s). The learned representations can boost performance in many network analysis tasks, e.g., graph classification [1], link prediction [14] and role discovery [29]. Graph embedding, according to the target, can be categorized into node-level and graph-level methods.

Previous node-level methods mainly viewed embedding as dimensionality reduction techniques [13]. With increasing attention attracted by neural network research, unsupervised neural network techniques have opened up a new world for embedding. word2vec as well as Skip-Gram and CBOW [26] learn low-rank representations of words in text based on word context and show promising results of different NLP tasks. Based on word2vec, DeepWalk [33] first introduces such embedding mechanism to networks by treating nodes as words and random walks as sentences. Afterwards, a sequence of studies have been conducted to improve DeepWalk either by extending the definition of neighborhood to higher-order proximities [34] or incorporating more information for node representations such as attributes [22] and heterogeneity [7].
Table 2: Results on the Small Network

\begin{tabular}{ccc}
\hline Methods & Precision & Recall \\
\hline noded2vec & 0.4512 & 0.4405 \\
graph2vec & 0.5634 & 0.5774 \\
NetLSD & 0.6756 & 0.7143 \\
Gl2vec & 0.5634 & 0.5774 \\
FGSD & 0.6756 & 0.7143 \\
GeoScattering & 0.7878 & 0.8512 \\
Sub2Vec & 0.7878 & 0.8512 \\
SADE (Ours) & $\mathbf{0 . 9 0 0 0}$ & $\mathbf{0 . 9 8 8 1}$ \\
\hline
\end{tabular}

Table 3: Results on the Large Network

\begin{tabular}{ccc}
\hline Methods & Precision & Recall \\
\hline noded2vec & 0.4045 & 0.4115 \\
graph2vec & 0.5086 & 0.5082 \\
NetLSD & 0.5387 & 0.5369 \\
Gl2vec & 0.5312 & 0.5297 \\
FGSD & 0.5134 & 0.5120 \\
GeoScattering & 0.4936 & 0.4939 \\
Sub2Vec & 0.5086 & 0.5082 \\
SADE (Ours) & $\mathbf{0 . 5 6 1 2}$ & $\mathbf{0 . 5 5 8 4}$ \\
\hline
\end{tabular}

On the other hand, different graph-level embedding methods have been proposed. graph2vec [27] first constructs WeisfeilerLehman tree features for nodes in graphs and then learn graph representations by decomposing the graph-feature co-occurrence matrix. NetLSD [35] extracts compact graph signatures based on the heat or wave kernel of the Laplacian, which inherit formal properties of the Laplacian spectrum. Gl2vec [8] extends graph2vec by further exploring the embedding of the defined line graphs, complementing the learning information of graph2vec. The learned embeddings are generated by concatenating the embeddings learned from graph2vec and the line graphs, aiming to preserve the missed edge features by graph2vec. FGSD [36] learns graph-level representations based on the discovery of family of graph spectral distances. GeoScattering [11] presents the geometric scattering transform as a deep filter bank for feature extraction, also proves its potential of serving as universal representations of graphs. Sub2Vec [1] also 
utilizes PV model [19] to learn distributed representations of subgraphs. It extends PV to learn subgraph embedding while preserving distance between subgraphs that have similar node co-occurrences reflected by paths of node id and degrees.

Recently, to bridge graph embedding and anomaly detection, learning anomaly aware network representations also attracts huge attentions [4, 23]. SEANO [23] is a semi-supervised network embedding approach which learns a low-dimensional vector representation that systematically captures the topological proximity, attribute affinity and label similarity of nodes. ONE [4] jointly aligns and optimizes the structures and attributes to generate robust network embeddings by minimizing the effects of outlier nodes. However, these studies only focus on node-level anomaly but our framework aims at detecting subgraph-level anomaly.

\section{CONCLUSIONS}

In this paper, we introduced a novel problem of detecting subgraphlevel anomalies in financial transaction networks whose structures deviate from underlying majority subgraphs in the network. To capture the local and global structural similarities between subgraphs, we proposed a role-guided anomaly detection framework (SADE) which allows learning the role-guided subgraph embedding in which state-of-art anomaly detection approaches can be used effectively. Our extensive experiments on transaction networks demonstrated the effectiveness of SADE in detecting anomalous subgraphs with the use of the Isolation Forest in the learnt embedding. This promising results suggest that SADE can be employed in the early warning systems at financial institutions.

\section{ACKNOWLEDGMENTS}

This work is partially supported by the Dutch Research Council (NWO).

\section{REFERENCES}

[1] Bijaya Adhikari, Yao Zhang, Naren Ramakrishnan, and B Aditya Prakash. 2018 Sub2vec: Feature learning for subgraphs. In Pacific-Asia Conference on Knowledge Discovery and Data Mining. Springer, 170-182.

[2] Edoardo M Airoldi, David M Blei, Stephen E Fienberg, and Eric P Xing. 2008 Mixed membership stochastic blockmodels. Fournal of Machine Learning Research 9, Sep (2008), 1981-2014.

[3] Archana Anandakrishnan, Senthil Kumar, Alexander Statnikov, Tanveer Faruquie, and Di Xu. 2018. Anomaly detection in finance: editors' introduction. In KDD 2017 Workshop on Anomaly Detection in Finance. 1-7.

[4] Sambaran Bandyopadhyay, N Lokesh, and M Narasimha Murty. 2019. Outlier aware network embedding for attributed networks. In Proceedings of the AAAI Conference on Artificial Intelligence, Vol. 33. 12-19.

[5] Monowar H Bhuyan, Dhruba Kumar Bhattacharyya, and Jugal K Kalita. 2013 Network anomaly detection: methods, systems and tools. Ieee communications surveys \& tutorials 16, 1 (2013), 303-336.

[6] Raghavendra Chalapathy, Edward Toth, and Sanjay Chawla. 2018. Group anomaly detection using deep generative models. In foint European Conference on Machine Learning and Knowledge Discovery in Databases. Springer, 173-189.

[7] Shiyu Chang, Wei Han, Jiliang Tang, Guo-Jun Qi, Charu C Aggarwal, and Thomas S Huang. 2015. Heterogeneous network embedding via deep architectures. In Proceedings of the 21th ACM SIGKDD International Conference on Knowledge Discovery and Data Mining. ACM, 119-128.

[8] Hong Chen and Hisashi Koga. 2019. GL2vec: Graph Embedding Enriched by Line Graphs with Edge Features. In International Conference on Neural Information Processing. Springer, 3-14.

[9] Kaize Ding, Jundong Li, Rohit Bhanushali, and Huan Liu. 2019. Deep anomaly detection on attributed networks. In Proceedings of the 2019 SIAM International Conference on Data Mining. SIAM, 594-602.

[10] Andrew Elliott, Mihai Cucuringu, Milton Martinez Luaces, Paul Reidy, and Gesine Reinert. 2019. Anomaly detection in networks with application to financial transaction networks. arXiv preprint arXiv:1901.00402 (2019).

[11] Feng Gao, Guy Wolf, and Matthew Hirn. 2019. Geometric Scattering for Graph Data Analysis. In International Conference on Machine Learning. 2122-2131.

[12] Jing Gao, Feng Liang, Wei Fan, Chi Wang, Yizhou Sun, and Jiawei Han. 2010. On community outliers and their efficient detection in information networks. In Proceedings of the 16th ACM SIGKDD international conference on Knowledge discovery and data mining. 813-822.

[13] Palash Goyal and Emilio Ferrara. 2018. Graph embedding techniques, applications, and performance: A survey. Knowledge-Based Systems 151 (2018), 78-94.

[14] Aditya Grover and Jure Leskovec. 2016. node2vec: Scalable feature learning for networks. In Proceedings of the 22nd ACM SIGKDD international conference on Knowledge discovery and data mining. 855-864.

[15] Leonardo Gutiérrez-Gómez, Alexandre Bovet, and Jean-Charles Delvenne. 2019. Multi-scale Anomaly Detection on Attributed Networks. arXiv preprint arXiv:1912.04144 (2019).

[16] Keith Henderson, Brian Gallagher, Tina Eliassi-Rad, Hanghang Tong, Sugato Basu, Leman Akoglu, Danai Koutra, Christos Faloutsos, and Lei Li. 2012. Rolx: structural role extraction \& mining in large graphs. In Proceedings of the 18th ACM SIGKDD international conference on Knowledge discovery and data mining. $1231-1239$.

[17] Keith Henderson, Brian Gallagher, Lei Li, Leman Akoglu, Tina Eliassi-Rad, Hanghang Tong, and Christos Faloutsos. 2011. It's who you know: graph mining using recursive structural features. In Proceedings of the 17th ACM SIGKDD international conference on Knowledge discovery and data mining. 663-671.

[18] Ramakrishnan Kannan, Hyenkyun Woo, Charu C Aggarwal, and Haesun Park. 2017. Outlier detection for text data. In Proceedings of the 2017 siam international conference on data mining. SIAM, 489-497.

[19] Quoc Le and Tomas Mikolov. 2014. Distributed representations of sentences and documents. In International conference on machine learning. 1188-1196.

[20] Daniel D Lee and H Sebastian Seung. 2001. Algorithms for non-negative matrix factorization. In Advances in neural information processing systems. 556-562.

[21] Jundong Li, Harsh Dani, Xia Hu, and Huan Liu. 2017. Radar: Residual Analysis for Anomaly Detection in Attributed Networks.. In I7CAI. 2152-2158.

[22] Jundong Li, Harsh Dani, Xia Hu, Jiliang Tang, Yi Chang, and Huan Liu. 2017. Attributed network embedding for learning in a dynamic environment. In Proceedings of the 2017 ACM on Conference on Information and Knowledge Management. 387-396.

[23] Jiongqian Liang, Peter Jacobs, Jiankai Sun, and Srinivasan Parthasarathy. 2018. Semi-supervised embedding in attributed networks with outliers. In Proceedings of the 2018 SIAM International Conference on Data Mining. SIAM, 153-161.

[24] Fei Tony Liu, Kai Ming Ting, and Zhi-Hua Zhou. 2008. Isolation forest. In 2008 Eighth IEEE International Conference on Data Mining. IEEE, 413-422.

[25] Fei Tony Liu, Kai Ming Ting, and Zhi-Hua Zhou. 2012. Isolation-based anomaly detection. ACM Transactions on Knowledge Discovery from Data (TKDD) 6, 1 (2012), 1-39.

[26] Tomas Mikolov, Ilya Sutskever, Kai Chen, Greg S Corrado, and Jeff Dean. 2013. Distributed representations of words and phrases and their compositionality. In Advances in neural information processing systems. 3111-3119.

[27] Annamalai Narayanan, Mahinthan Chandramohan, Rajasekar Venkatesan, Lihui Chen, Yang Liu, and Shantanu Jaiswal. 2017. graph2vec: Learning distributed representations of graphs. arXiv preprint arXiv:1707.05005 (2017).

[28] Yulong Pei. 2020. On local and global graph structure mining. Number 2020-05 in SIKS Dissertation Series. Technische Universiteit Eindhoven.

[29] Yulong Pei, Xin Du, Jianpeng Zhang, George Fletcher, and Mykola Pechenizkiy. 2020. struc2gauss: Structural role preserving network embedding via Gaussian embedding. Data Mining and Knowledge Discovery (2020).

[30] Yulong Pei, Jianpeng Zhang, George HL Fletcher, and Mykola Pechenizkiy. 2018. DyNMF: Role Analytics in Dynamic Social Networks.. In IfCAI. 3818-3824.

[31] Zhen Peng, Minnan Luo, Jundong Li, Huan Liu, and Qinghua Zheng. 2018. ANOMALOUS: A Joint Modeling Approach for Anomaly Detection on Attributed Networks.. In IFCAI. 3513-3519.

[32] Bryan Perozzi and Leman Akoglu. 2016. Scalable anomaly ranking of attributed neighborhoods. In Proceedings of the 2016 SIAM International Conference on Data Mining. SIAM, 207-215.

[33] Bryan Perozzi, Rami Al-Rfou, and Steven Skiena. 2014. Deepwalk: Online learning of social representations. In Proceedings of the 20th ACM SIGKDD international conference on Knowledge discovery and data mining. ACM, 701-710.

[34] Jian Tang, Meng Qu, Mingzhe Wang, Ming Zhang, Jun Yan, and Qiaozhu Mei. 2015. Line: Large-scale information network embedding. In Proceedings of the 24th International Conference on World Wide Web. International World Wide Web Conferences Steering Committee, 1067-1077.

[35] Anton Tsitsulin, Davide Mottin, Panagiotis Karras, Alexander Bronstein, and Emmanuel Müller. 2018. Netlsd: hearing the shape of a graph. In Proceedings of the 24th ACM SIGKDD International Conference on Knowledge Discovery \& Data Mining. 2347-2356.

[36] Saurabh Verma and Zhi-Li Zhang. 2017. Hunt for the unique, stable, sparse and fast feature learning on graphs. In Advances in Neural Information Processing Systems. 88-98. 